OPEN ACCESS

Edited by:

Xin Tian,

First Affiliated Hospital of Chongqing

Medical University, China

Reviewed by:

Tatsuro Misu,

Tohoku University, Japan

Moussa Antoine Chalah,

Hôpitaux Universitaires Henri Mondor,

France

${ }^{*}$ Correspondence:

Zhong Chen

chenzhong@zju.edu.cn

Meiping Ding

dmp-neurology@zju.edu.cn

Specialty section:

This article was submitted to

Multiple Sclerosis and

Neuroimmunology,

a section of the journal

Frontiers in Neurology

Received: 06 October 2018 Accepted: 17 December 2018

Published: 10 January 2019

Citation:

Zheng Y, Zhang Y, Cai M, Lai N, Chen $Z$ and Ding M (2019) Central

Nervous System Involvement in ANCA-Associated Vasculitis: What

Neurologists Need to Know.

Front. Neurol. 9:1166

doi: 10.3389/fneur.2018.01166

\section{Central Nervous System Involvement in ANCA-Associated Vasculitis: What Neurologists Need to Know}

\author{
Yang Zheng ${ }^{1}$, Yinxi Zhang ${ }^{1}$, Mengting Cai ${ }^{1}$, Nanxi Lai ${ }^{2}$, Zhong Chen ${ }^{1,2 *}$ and Meiping Ding ${ }^{1 *}$ \\ ${ }^{1}$ Department of Neurology, Second Affiliated Hospital, School of Medicine, Zhejiang University, Hangzhou, China, \\ ${ }^{2}$ Department of Pharmacology, Key Laboratory of Medical Neurobiology of the Ministry of Health of China, College of \\ Pharmaceutical Sciences, School of Medicine, Zhejiang University, Hangzhou, China
}

Objective: To provide a comprehensive review of the central nervous system (CNS) involvement in anti-neutrophil cytoplasmic antibody (ANCA)-associated vasculitis (AAV), including the pathogenesis, clinical manifestations, ancillary investigations, differential diagnosis, and treatment. Particular emphasis is placed on the clinical spectrum and diagnostic testing of AAV.

Recent Findings: AAV is a pauci-immune small-vessel vasculitis characterized by neutrophil-mediated vasculitis and granulomatousis. Hypertrophic pachymeninges is the most frequent CNS presentation. Cerebrovascular events, hypophysitis, posterior reversible encephalopathy syndrome (PRES) or isolated mass lesions may occur as well. Spinal cord is rarely involved. In addition, ear, nose and throat (ENT), kidney and lung involvement often accompany or precede the CNS manifestations. Positive ANCA testing is highly suggestive of the diagnosis, with each ANCA serotype representing different groups of AAV patients. Pathological evidence is the gold standard but not necessary. Once diagnosed, prompt initiation of induction therapy, including steroid and other immunosuppressants, can greatly mitigate the disease progression.

Conclusions and Relevance: Early recognition of AAV as the underlying cause for various CNS disorders is important for neurologists. Ancillary investigations especially the ANCA testing can provide useful information for diagnosis. Future studies are needed to better delineate the clinical spectrum of CNS involvement in AAV and the utility of ANCA serotype to classify those patients.

Evidence Review: We searched Pubmed for relevant case reports, case series, original research and reviews in English published between Sep 1st, 2001 and Sep 1st, 2018. The following search terms were used alone or in various combinations: "ANCA," "proteinase 3/PR3-ANCA," "myeloperoxidase/MPO-ANCA," "ANCA-associated vasculitis," "Wegener's granulomatosis," "microscopic polyangiitis," "Central nervous system," "brain" and "spinal cord". All articles identified were full-text papers.

Keywords: anti-neutrophil cytoplasmic antibodies, vasculitis, granulomatosis with polyangiitis, microscopic polyangiitis, central nervous system 


\section{INTRODUCTION}

Central nervous system (CNS) vasculitis, with its myriad and evolving presentations, always poses a great diagnostic challenge for neurologists. It occurs either as part of a systemic vasculitis, or a primary disorder restricted to the CNS (1). Anti-neutrophil cytoplasmic antibody (ANCA) associated vasculitis (AAV), a systemic small-vessel vasculitis, is characterized by pathogenic ANCA production (1). In the clinical practice, AAV mainly includes granulomatosis with polyangiitis (GPA), microscopic polyangiitis (MPA) and eosinophilic granulomatosis with polyangiitis (EGPA) (1). Timely recognition and diagnosis of AAV is important, since the progressive disease can be dramatically mitigated by prompt use with steroid and other immunosuppressive agents.

Neurologic involvement is not uncommon in AAV throughout the disease course, ranging from 22 to $54 \%$ in patients with GPA (2-5) and 34 to $72 \%$ in those with MPA (2-4). CNS is affected in $<15 \%$ of patients with AAV (5) but accounts for much of the morbidity in those patients $(1-3,7-10)$. However, the heterogeneous CNS symptoms in AAV may hinder early diagnosis among neurologists, causing treatment delays and disease progression, leading to relapses, or even death.

Therefore, this review aims to increase the awareness of AAV among neurologists. We mainly focus on GPA and MPA, which have distinctive features compared with EGPA (610). This review comprehensively illustrates the pathogenesis, CNS manifestations, ancillary investigations, and treatment algorithms warranted for AAV patients with CNS involvement. In particular, we put a special emphasis on its clinical spectrum and the utility of ACNA testing in diagnosing and subtyping those patients.

\section{PATHOPHYSIOLOGY}

A basic understanding of how pathogenic ANCAs are induced, take effect and invade the CNS helps to understand its manifestations and illuminate potential targets for treatment in AAV. Pathogenic ANCAs, targeting mainly at proteinase 3 (PR3) and myeloperoxidase (MPO) expressed by innate immune cells, are the major contributor to the pathogenesis of $\mathrm{AAV}$, according to in vitro and in vivo experimental data (11). An overview of the pathophysiology is shown in Figure 1. Pathogenic ANCAs are induced by the interplay of multiple environmental, genetic, and immunological factors $(8,11,15)$. An encounter with the antisense peptides of PR3 or MPO triggers the immunological self-amplication network (11). The antigen-recognition capability of each individual, however, is more likely genetically determined (8). In addition, the generation of pathogenic ANCAs is further facilitated by an impaired immunological regulation, as in the pathogenesis of the few treatable neurological disorders (16-19). The function of regulatory $\mathrm{T}\left(\mathrm{T}_{\mathrm{REG}}\right)$ cells and regulatory $\mathrm{B}$ cells with $\mathrm{CD} 5$ expression are suppressed, whereas the circulating effector memory T cells ( $\left.\mathrm{T}_{\mathrm{EM}}\right)$ (20) and ANCA-producing B cells (15) are proliferated and activated.
Following the generation of pathogenic ANCAs, different pathways lead to the two major pathological changes in AAV, namely vasculitis, and granulomatosis. Neutrophils, activated by pathogenic ANCAs and fueled by the alternative complement pathway (13), play the central role. Activated neutrophils can transmigrate the vessel wall and undergo respiratory burst, degranulation, neutrophil extracellular traps (NETs), apoptosis and necrosis (14), causing disruptions of the endothelium and thus activation of the coagulation cascade, leading to fibroid necrosis at sites of vasculitic inflammation. This neutrophilactivation process is further augmented by the complement system, especially the alternative pathway, with C5a playing a key role in-between (13). By contrast, the pathogenesis of extravascular granulomatosis is less well-understood. Current thinking holds that the chronic inflammation is initiated by the acute neutrophil-mediated necrosis (21). Subsequently, defects in the cell death machinery and aberrant reaction of monocytes and macrophages contribute to the chronic inflammation and granulomatosis formation in AAV.

CNS can be affected in AAV through one of the following pathways $(22,23)$ : (1) inflammation, obstruction or increased permeability of the small to medium-sized cerebral vessels due to systemic vasculitis; (2) infiltration or compression of granulomatous pathology from adjacent structures; (3) granulomatous lesions developing de novo within the CNS. Mechanisms vary according to the specific CNS structures involved. In general, extra-axial lesions involving the dura, or pituitary gland are mainly attributed to granulomatous inflammation, while parenchyma pathologies are mediated by vasculitis and breakdown of blood brain barrier (24). However, it remains unclear whether pathogenic ANCAs are produced intrathecally or from the systemic circulation and how the two ANCA serotypes contribute to different CNS manifestations.

\section{THE MANY FACES OF AAV WITH CNS INVOLVEMENT}

\section{Epidemiology}

AAV, especially GPA and MPA, is a multisystem disease. Up to $40-50 \%$ of patients have a remitting-relapsing course $(7,12)$. The onset of CNS flare is mostly acute or subacute, depending on the specific neurological syndrome. CNS symptoms usually present late in the disease course $(5,25)$. No gender predilection is observed and most patients tend to have their first CNS flare in the middle age $(5,25,26)$.

\section{Overview of Systemic AAV}

It is of great help to know the accompanying systemic symptoms at the time of or prior to CNS flare. A longterm history of constitutional symptoms like fever, weight loss, fatigue and arthralgia are often suggestive of an autoimmune etiology. In addition, organs susceptible to AAV damage include ear, nose and throat (ENT), lung and kidney (6). ENT involvement for over 3 months, presenting as chronic sinusitis, otitis media, or mastoiditis, is diagnostic of AAV, $(3,7,24)$. History of renal dysfunction (proteinuria, hematuria 


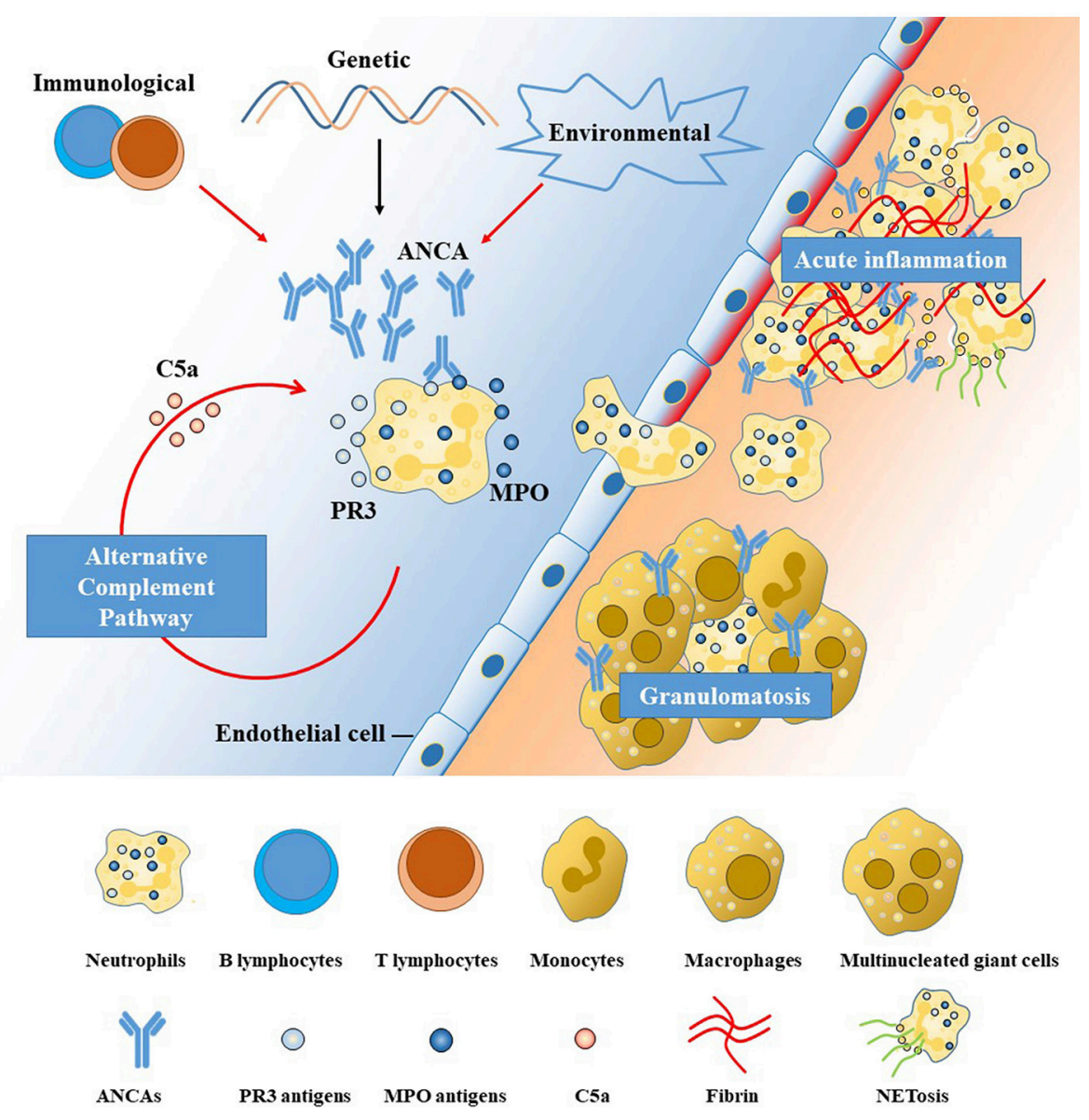

FIGURE 1 | Pathogenesis of anti-neutrophil cytoplasmic antibody (ANCA)-associated vasculitides (AAV). The left side of the diagram (with blue background) represents the blood stream and the right (with orange background) the interstitial tissue, separated by a line of endothelial cells. ANCAs are autoantibodies directed against proteins in the cytoplasmic granules of neutrophils. The two antigenic targets are proteinase 3 (PR3) and myeloperoxidase (MPO) normally expressed on the surface or inside the cytoplasm of resting neutrophils $(11,12)$. The interplay among genetic, environmental, and immunological factors contributes to the high membrane expression and release of PR3 and MPO, leading to the production and proliferation of pathogenic ANCAs. Primed neutrophils are activated by ANCAs and transmigrate the vessel wall, undergoing respiratory bursts, degranulation, and neutrophil extracellular traps (NETs) generation (11), which are further augmented by the alternative complement pathway (13). The neutrophil-mediated processes are the major contributor to the injury and inflammation of the endothelial cells lining the vascular wall in the early phase (14). Monocytes are subsequently recruited at sites of acute inflammation and necrosis, inducing the development of granulomatous inflammation mainly mediated by an exaggerated monocyte/macrophage reaction (11). Potential treatment targets are illustrated by red arrows in the figure, including the T-cell and B-cell dysregulation, environmental triggers (microbes, drugs), aberrant activation of alternative complement pathway and NETs. ANCA, anti-neutrophil cytoplasmic antibody; MPO, myeloperoxidase; PR3, proteinase 3; NET, neutrophil extracellular trap.

and acute kidney failure) and pulmonary problems (pulmonary nodules, infiltration, alveolar hemorrhage, chronic cough, asthma, or rarely respiratory failure) were also frequently reported. Therefore, for patients suspected with AAV, an investigation of systemic symptoms is warranted. Manifestations highly suggestive of AAV are listed in Table 1.

\section{Clinical and Imaging Spectrum of CNS Involvement in AAV}

CNS presentations in AAV patients vary, including headache, ischemic infarction, intracranial hemorrhage, encephalopathy (seizures, neuropsychiatric disorders, confusion, or altered consciousness), and rarely spinal cord symptoms. Those symptoms are caused by the involvement of corresponding CNS structures including the dura mater, brain parenchyma, pituitary gland, spinal cord, and leptomeninges with decreasing frequency.

\section{Brain Parenchyma Involvement}

\section{(a) Cerebrovascular events}

Ischemic infarctions and intracranial hemorrhages, though rare, can be the initial presentation of $\mathrm{AAV}$ and are always associated with significant morbidity (29-31). Timely recognition of AAV as the underlying cause is difficult at the first visit in the emergency room due to its rarity. Therefore, the distinguishing features of AAVrelated strokes can aid in early diagnosis and improve 
TABLE 1 | Systemic manifestations of AAV other than CNS\#

\begin{tabular}{|c|c|}
\hline Involved organ & Manifestations \\
\hline Constitutional symptoms & Fever, weight loss, polyarthralgia, polymyalgia, malaise, polyarthritis \\
\hline Ear nose and throat (ENT) & Chronic sinusitis*, chronic otitis media*, chronic mastoiditis*, bloody nasal discharge/crusts/ulcers/granuloma*, subglottic stenosis* \\
\hline Trachea and lung & Hemoptysis (due to pulmonary hemorrhage* or tracheobronchial disease), lung nodules*, cough, dyspnea, pleuritic pain \\
\hline Kidney & Glomerulonephritis (especially rapidly progressive glomerulonephritis)* \\
\hline Skin & Leukocytoclastic angiitis* (typically palpable purpura involving the lower extremities with focal necrosis and ulceration) \\
\hline Eye and orbit & Epislceritis/scleritis ${ }^{\star}$, uveitis, conjunctivitis, corneal ulceration, retinal vasculitis, optic neuropathy, retro-orbital mass or inflammation* \\
\hline Peripheral nervous system & Mononeuritis multiplex*, cranial neuropathies* \\
\hline Gastrointestional tract & Diarrhea, nausea, vomiting, abdominal pain, gastrointestinal hemorrhage; Elevated liver enzymes \\
\hline Cardiovascular & Ischemic cardiac pain, cardiomyopathy, congestive heart failure, loss of pulses, valvular heart disease, pericarditis \\
\hline
\end{tabular}

\# The table is adapted from previous reviews on related topics $(6,12,27)$.

${ }^{*}$ Clinical features highly suggestive of $\operatorname{AAV}(6,28)$.

$A A V$, anti-neutrophil cytoplasmic antibody-associated vasculitis; CNS, central nervous system.

prognosis. Ischemic infarctions typically present as an isolated or multiple lesions affecting the white matter, since distal penetrating vessels are predominantly affected. Medullary and pontine infarctions were also reported in some cases $(29,30)$. Ischemic infarctions caused by AAV are typically resistant to antiplatelet therapy and tend to recur without proper immunosuppressive therapy. Patients with AAV are also at an increased risk of hemorrhagic transformation after reperfusion therapy of ischemic stroke (32). Hemorrhagic events occur less often. They more often affect the brain parenchyma (31), and sometimes the subarachnoid space (33). Brain Imaging findings usually correspond to the specific disease in each patient, involving ischemic, hemorrhagic lesions, or variable degrees of smallvessel diseases affecting both white and gray matter (34). Nonspecific white matter lesions with T2 hyperintensities can appear in the periventricular, subcortical regions, the basal ganglia, the mesencephalon and pons.

(b) Posterior reversible encephalopathy syndrome

Posterior reversible encephalopathy syndrome (PRES) is a rare yet unique complication in the late phase of $\mathrm{AAV}$ (35, 36). Clinically, this entity typically presents with an acute onset. Symptoms are generalized and include encephalopathy, seizures, headache and visual disturbance. Brain imaging typically reveals findings consistent with vasogenic edema, predominantly involving the bilateral parieto-occipital regions. Most patients with PRES have a dramatic improvement within days to weeks, with only the supportive therapy. Symptoms may recur when the underlying AAV is not well-controlled.

(c) Isolated parenchymal mass lesions

Isolated parenchymal mass lesions were very rarely reported in AAV (37-39), which often present with a discrete granuloma. Symptoms vary depending on the location of lesions, with seizures as the most frequent presentation (38). Brain magnetic resonance imaging (MRI) of isolated parenchymal granulomas reveals a well-delineated mass with a high signal intensity on T2-weighted images and enhancement on gadolinium-enhanced sequences (39). (d) Cognitive impairment

Cognitive decline, though mostly subclinical and mild, can occur in AAV patients as well, with an estimated prevalence of $30 \%$ (40). According to one study of 13 AAV patients (40), the pattern of cognitive impairment, mainly affecting abstract reasoning, attention and non-verbal memory, is different from that of age-related dementia. On brain MRI, multiple white matter lesions, mainly located in the periventricular or juxtacortical areas, are often associated with the cognitive impairment (40).

\section{Brain Meninges Involvement}

Pachymeninges is affected more frequently than leptomeninges (41). The frequency of hypertrophic pachymeningitis (HP) in adult AAV ranges from 18 to $35 \%$, depending on the methodology of studies and the sample populations selected (41, 42). Manifestations of AAV-related pachymeningitis vary depending on the location and extent of inflammation. Headache, the dominant symptom of HP, is often severe and resistant to analgesics (22). Besides, neck stiffness is not commonly seen along with headache. Cranial nerves may be compressed by the thickened dura mater and cause symptoms such as visual loss, double vision, and facial palsies. Other times the pachymeningeal inflammation may infiltrate the brain parenchyma and cause impaired consciousness and seizures (24, 42). Notably, an entity named "CNS-limited AAV" was recently proposed by Yoloseki et al. for patients with MPO-ANCApositive hypertrophic pachymeningitis, characterized by an elderly female predominance, less severe neurological damages, and lower rates of developing into the generalized disease (24). Yet it remains unclear whether the "CNS-limited AAV" represents a novel AAV phenotype or merely a transient disease stage (24).

Imaging studies are valuable in identifying hypertrophic thickening of the dura mater, monitoring disease activity and assessing the damage of adjacent structures. On brain MRI and computed tomography (CT) scans, HP typically shows linear thickening of dura mater or a bulging mass with 
enhancement. Gadolinium-enhanced T1-weighted MR imaging offers certain advantages over other sequences in identifying active inflammation of the thickened dura mater with superior spatial resolution. The brain pachymeningitis can involve the tentorium cerebelli, cranial fossa, cavernous sinus, falx cerebri, or convexity with no site preference. Additionally, brain-imaging studies can help assess the potential sinonasal and orbital involvement, a common occurrence in AAV. Additionally, the damage of adjacent bone structures is prominent on brain CT scans $(41,43)$.

\section{Pituitary Gland and Stalk Involvement}

Inflammation of the pituitary, termed hypophysitis, is rarely seen in AAV, yet requiring special consideration (44-46). Constitutional symptoms including fatigue, lethargy, headache, weight loss, and appetite loss frequently occur. Endocrine disturbances most commonly include diabetes insipidus, and hypogonadism (45). Other less common ones include hypothyroidism, adrenocorticotropic hormone (ACTH) deficiency and growth hormone (GH) deficiency $(45,47)$. Pituitary stalk compression resulting from pituitary enlargement may cause hyperprolectinemia as well (22). Visual deficits result with optic chiasm compression (45). Outcome of hypopituitarism is less favorable, despite the treatment with immunosuppressive agents. Pituitary dysfunction tends to persist, even though other systemic symptoms can come to remission after standard treatment $(45,46)$.

Brain MRI typically shows an enlarged pituitary gland or thickened stalk with peripheral enhancement on post-contrast sequences. Other findings include the lack of posterior pituitary hyperintensity on T1 images. Normal MRI, as reported in some cases, does not exclude pituitary involvement (47).

\section{Spinal Cord Involvement}

In general, spinal cord is rarely involved in AAV (48). The three possible mechanisms underlying spinal cord involvement include necrotizing inflammation of the spinal vasculature, compression of the spinal cord by inflamed thickened meninges as well as the formation of primary spinal granulomas (48). Clinical syndromes of the spinal cord include hypertrophic pachymeningitis $(41,48)$ and compressive myelopathy (49). Contrast-enhanced MRI of the spinal cord is of great diagnostic value and biopsy is often warranted to confirm the diagnosis.

\section{Non-CNS Entities Highly Associated With CNS Involvement}

(a) Cranial neuropathy

Cranial neuropathies are rarely the only manifestation in AAV, but rather coexist with other CNS symptoms such as headache and systemic symptoms. Previous investigations found a prevalence between 2 and $10 \%$ of cranial neuropathies in patients diagnosed with GPA (50) and MPA $(51,52)$. Commonly affected cranial nerves in AAV include cranial nerve II-VIII (50). Bulbar palsy, by contrast, is less commonly involved (53).

\section{(b) Orbital disease}

Eye involvement was reported in around $30-50 \%$ of patients diagnosed with GPA (54), with the orbit and sclera most frequently involved. MPA, by contrast, rarely impair eye movement (52). Symptoms vary and typically include proptosis, diplopia, decreased vision and orbital pain (55), resulting from compressions of ocular granuloma, granulomatous inflammation of the optic nerve and ischemic optic neuropathy due to vascular occlusion (56). MR imaging and CT can help reveal mucosal and bony lesions in the orbit and rule out continuous extension.

(c) Parasinal disease

For patients with CNS manifestations suspected of AAV, an evaluation of paranasal sinuses is a must. Findings indicative of granulomatosis in the paranasal sinuses include a softtissue mass, thickening of the sinus wall, or "ground-glass" material in the lumen (34). As in orbital involvement, noncontrast CT scan has its diagnostic value in evaluating the nasal cavity, paranasal sinuses, mastoids and temporal bone, due to a better resolution of mucosal and bony pathologies compared with MRI (43). Brain MRI may also aid in the evaluation of soft tissue masses within those cavities.

\section{Differences Between ANCA Serotypes}

There is an increasing consensus that the ANCA serotype, either MPO-ANCA or PR3-ANCA, has a crucial role in AAV (12). Distinctively, previous studies revealed that the ANCA serotype represents two separate groups of patients in epidemiology, genetic background, clinical features, laboratory features as well as prognosis. We summarized the differences between MPOand PR3-ANCA positive AAV in Table 2. Specifically, for AAV patients with CNS involvement, the ANCA serotype matters as well (24). Though the two serotypes tended to affect the nervous system with a similar frequency (57), they differ in the pattern and severity of CNS involvement. According to one study of patients with HP in Japan, MPO-ANCA-positive HP had an elderly female predominance, more frequently had lesions limited to the dura mater and upper airways, and was less likely to have a generalized systemic progression. On the contrary, PR3-ANCA-positive HP tended to have more severe neurological damages and a generalized disease progression (24). However, evidence remains limited to patients with HP. Extrapolation of the utility of ANCA serotype in other CNS manifestations remains to be further studied.

\section{DIAGNOSIS}

The diagnosis of CNS involvement in AAV, similar to the disease overall, requires consideration of clinical, serological, radiographic, and, when available, pathological evidence (61). In patients with established AAV, new-onset neurologic deficits with abnormal radiological and cerebrospinal fluid (CSF) findings are suggestive of CNS involvement. For patients without established underlying disease, neurologic symptoms closely compatible with CNS syndromes known to arise in AAV warrant further investigations for the systemic disease. In either case, ancillary tests are of important value in making the 
TABLE 2 | Comparison of PR3- and MPO-ANCA-positive AAV.

\begin{tabular}{|c|c|c|}
\hline Features & PR3-ANCA-positive AAV & MPO-ANCA-positive AAV \\
\hline Pathophysiology $(12,57)$ & $\begin{array}{l}\text { Apoptosis of endothelial cells; Release of sFlt1 by monocytes; } \\
\text { No established mouse model }\end{array}$ & $\begin{array}{l}\text { Production of intracellular oxidants; No induction of release of } \\
\text { sFlt1 by monocytes; } \\
\text { Pathogenicity of autoantibody proved in mouse models }\end{array}$ \\
\hline Epidemiology $(12,57)$ & Northern Europe, America and Australia & Southern Europe and Asia \\
\hline Genetic background $(8,12,57)$ & HLA-DP, SERPINA1, PRTN3 & HLA-DQ, CTLA4 \\
\hline Clinical features $(12,57-59)$ & $\begin{array}{l}\text { More ENT involvement; } \\
\text { Cavititating pulmonary lesions, nodules and masses; More } \\
\text { organs involved }\end{array}$ & $\begin{array}{l}\text { More often renal-limited; } \\
\text { Fibrosing pulmonary lesions and patchy infiltrates }\end{array}$ \\
\hline Pathological features (12) & Granuloma and vasculitis & Granuloma and fibrosis \\
\hline Response to induction therapy $(12,60)$ & Better response to rituximab than cyclophosphamide & Increased risk of initial treatment failure \\
\hline Long-term outcome (12) & $\begin{array}{l}\text { More relapses } \\
\text { Better prognosis }\end{array}$ & $\begin{array}{l}\text { Less relapses } \\
\text { Higher risk of long-term kidney and alveolar damage } \\
\text { Worse prognosis }\end{array}$ \\
\hline
\end{tabular}

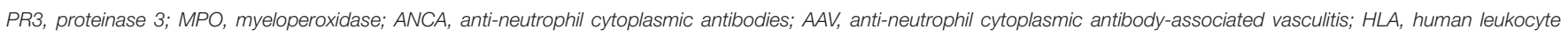
antigen; ENT, ear, nose and throat.

Box 1 | Investigations in AAV-related CNS involvement.

ANCA immunoassays (MPO- and PR3- ANCA testing)

CBC, CMP, CRP, ESR (to evaluate the organ functions and the state of inflammation)

Autoimmune panel, complement levels (to differentiate from other inflammatory conditions)

Endocrine panel (to evaluate the pituitary function)

Screening for HIV, hepatitis, and tuberculosis

Analysis of cerebrospinal fluid (measure inflammatory mediators and degradation proteins, assess the blood-brain barrier and exclude infection) Chest CT

Brain imaging (CT; MRI, suggested sequences include T1/T2 weighted-

imaging, FLAIR, DWI, SWI and contrast enhanced T1 sequences)

Biopsy

AAV, anti-neutrophil cytoplasmic antibody-associated vasculitis; ANCA, antineutrophil cytoplasmic antibodies; MPO, myeloperoxidase; PR3, proteinase 3; CBC, complete blood count; CMP, comprehensive metabolic panel; CRP, C reactive protein; ESR, erythrocyte sedimentation rate; HIV human immunodeficiency virus; CT, computed tomography; MRI, magnetic resonance imaging; FLAIR, fluid-attenuating inversion recovery; DWI, diffusion-weighted imaging; SWI, susceptibility-weighted imaging.

diagnosis and excluding other causes of the CNS abnormalities (Box 1). This section describes workup that can aid in the diagnosis of AAV with CNS involvement, and with special interest, pays extra attention to the value of ANCA testing in-between.

\section{ANCA Serology}

ANCA testing is strongly recommended for patients with clinical features suggestive of AAV (Table 1) $(6,62)$. Its value lies mainly in AAV screening, and when positive, warrants further investigations for confirmative diagnosis. Positivity of the test is highly suggestive of AAV but not diagnostic by itself (6). However, the combination of ANCA positivity and certain clinical features are sufficient for AAV diagnosis, according to the widely-used classification algorithm proposed by Watts (28). A negative ANCA immunoassay, which occurs in up to half of pathologically diagnosed GPA and MPA, does not exclude AAV $(6,63)$. The negativity may be related to the limited sensitivity of ANCA testing, especially at the early stage of disease without systemic involvement (7). The diagnosis of AAV cannot be excluded merely based on a negative ANCA test. Therefore, biopsy of the affected organ is required for seronegative patients (6).

Regarding the testing techniques of ANCA, the most recent consensus recommended high-quality antigen-specific immunoassays for MPO- and PR3- ANCAs detection (6). ELISAs are the preferred screening methodology with a high sensitivity and specificity. Indirect immunofluorescence (IIF) for cytoplasmic ANCA (C-ANCA) and perinuclear ANCA (P-ANCA) detection is no longer prioritized as the screening test, given its large variability and poor diagnostic accuracy (6). When necessary, performing another assay or testing with another different methodology can yield higher sensitivity and specificity (6). In cases of emergency such as pulmonary-renal syndrome, rapid screening assays for ANCAs, including dot blots and biochip technology, are also available (64).

Controversy remains in the role of ANCA levels in monitoring disease activity and prediction of prognosis (63). Published studies reveal inconsistent results regarding the role of ANCA levels in predicting clinical relapse and reflecting disease activity (65-67). A meta-analysis in 2012 concluded that both a rise in ANCA and persistently positive ANCA were strongly associated with disease relapse and had a modest predictive value (66). Furthermore, results from a recent single-center study indicated that the predictive value of ANCA was only significant in renalinvolved $\mathrm{AAV}$, compared with those without renal involvement (68). Overall, current thinking holds that ANCA levels alone are helpful but not sufficient to determine relapse or reflect disease activity (6), and ANCA testing is not recommended to guide clinical decisions on treatment (61). Nevertheless, severe relapses are unlikely without elevated ANCA levels (69). The significance of serial monitoring remains to be proven. 


\section{Other Laboratory Investigations}

A diligent workup should be ordered for patients suspected of AAV, including complete blood count (CBC), complete metabolic profile (CMP), acute phase reactants, the autoimmune panel, the endocrine panel, the complement level and investigations for potential infections. For patients with endocrine dysfunction or abnormal pituitary on MRI, levels of pituitary hormones also need to be investigated. Laboratory tests typically show elevated markers of inflammation. Leukocytosis, thrombocytosis, normochromic normocytic anemia, elevated erythrocyte sedimentation rate (ESR) and C-reactive protein (CRP) values are indicative of the diagnosis $(3,7)$. Kidney functions, including serum creatinine and urinalysis, are warranted for the evaluation of renal injury. Regarding the autoimmune panel, autoantibodies other than ANCAs can be present (63), including antinuclear antibodies, rheumatoid factors, IgG4, anti-glomerular basement membrane antibodies and antiphospholipid antibodies, with unknown significance (63). Lastly, for differential diagnosis, investigations for potential infections (tuberculosis, human immunodeficiency virus, hepatitis) and complement levels are also required.

\section{Cerebrospinal Fluid}

Lumbar puncture is warranted for suspected patients as well. CSF analysis has a high sensitivity but low specificity in the diagnosis of AAV-related CNS disease. Most had mild lymphocytepredominant pleocytosis, elevated protein levels and normal glucose level $(7,25,37)$. Therefore, a normal CSF analysis makes the diagnosis of AAV less likely. Infectious etiology and flow cytometry for atypical cancer cells should be tailored to clinical manifestations and risk factors.

\section{Pathology}

Histopathology is the gold standard for diagnosis of small vessel vasculitis. Samples can be taken from affected organs, most commonly the kidney and the skin. Lung and nasal biopsies are rarely performed, limited by the high rate of false negatives. Brain biopsies can also be taken from corresponding structures including the dura, brain parenchyma and the overlying leptomeninges. Typically two types of pathological findings have been described: (1) necrotizing vasculitis affecting small to medium vessels; (2) granulomatosis with inflammatory cell infiltration (monocytes, plasma cells, eosinophils, and polymorphonuclear leukocytes) (41). Fibrinoid necrosis and edema were also detected in some cases. In patients of HP, fibrosis of the dura mater was always present as well $(24,41)$. A negative yield of biopsy, however, does not exclude the diagnosis of AAV due to its segmental nature of lesions.

\section{Differential Diagnosis}

AAV must be distinguished from ANCA-positive conditions where ANCA does not exert a direct role in pathophysiology (Box 2). Multiple conditions other than AAV show an elevated titer of serum ANCAs. Nonetheless, ANCAs do not mediate a direct pathogenic role, but rather indicate a chronic immune response of neutrophil cell death most times (63). Compared with the mimics, a high level and affinity of ANCAs and clinical
Box 2 | Conditions other than AAV with positive ANCA immunoassays\#.

Vasculitis of other causes

Anti-GBM disease, IgA-vasculitis*

Gastrointestinal diseases

Ulcerative colitis, Primary Sclerosing Cholangitis, Inflammatory liver disease Systemic inflammatory conditions

IgG4-related disease, Rheumatoid arthritis, Systemic lupus erythematous Infection

Infective endocarditis, Tuberculosis, HIV, Amoeba infection

Malignancies

Hematological neoplasia

Drugs

Hydralazine, Propylthiouracil, Levamisole, Minocycline, Cocaine.

\# The box is based upon previous reviews on related topics $(6,27)$. Serum ANCAs should be tested according to the algorithm proposed by the 2017 consensus on testing of ANCAs (6).

*IgA-vasculitis can show positive IgA-ANCAs in the active stage of disease, but IgG-ANCAs were rarely reported (27).

AAV, anti-neutrophil cytoplasmic antibody-associated vasculitis; ANCA, antineutrophil cytoplasmic antibodies; GBM, glomerular basement membrane; HIV, human immunodeficiency virus.

features suggestive of AAV are important clues for the correct diagnosis (6).

Differential diagnosis can be further narrowed down to entities with both similar CNS manifestations and ANCA positivity. These mainly include systemic inflammatory diseases (IgG4 related-disease, rheumatoid arthritis, systemic lupus erythematosus, Bechet syndrome), infective diseases (tuberculosis, human immunodeficiency virus) and malignancies (lymphoma).

In particular, diagnosis of AAV can be especially difficult at an early stage with only CNS presentations and a seronegative ANCA test, which may be an under-recognized common occurrence (24). Characteristics and diagnostic criteria of this entity remain to be elucidated. Differential diagnosis is therefore protean, varying according to the specific CNS manifestation of the patient. We suggest a thorough investigation of systemic involvement to exclude alternative causes including infection and malignancy. In particular, A continued monitoring of ANCA testing may be useful for those with idiopathic hypertrophic pachymeningitis (24). Most importantly, biopsy of the lesion is highly recommended in such cases to confirm the diagnosis.

\section{TREATMENT}

Treatment should be started in a timely manner for patients highly suspected of AAV, even in the absence of pathological evidence. Steroid is an essential part of therapy, with the addition of a well-chosen immunosuppressant critical to prevent relapses and achieve remission of CNS symptoms in the long run. The treatment algorithm is shown in Figure 2. There are two phases of treatment, namely remissioninduction and remission-maintenance. The choice of regimen in each phase depends mainly on the disease stage of the 


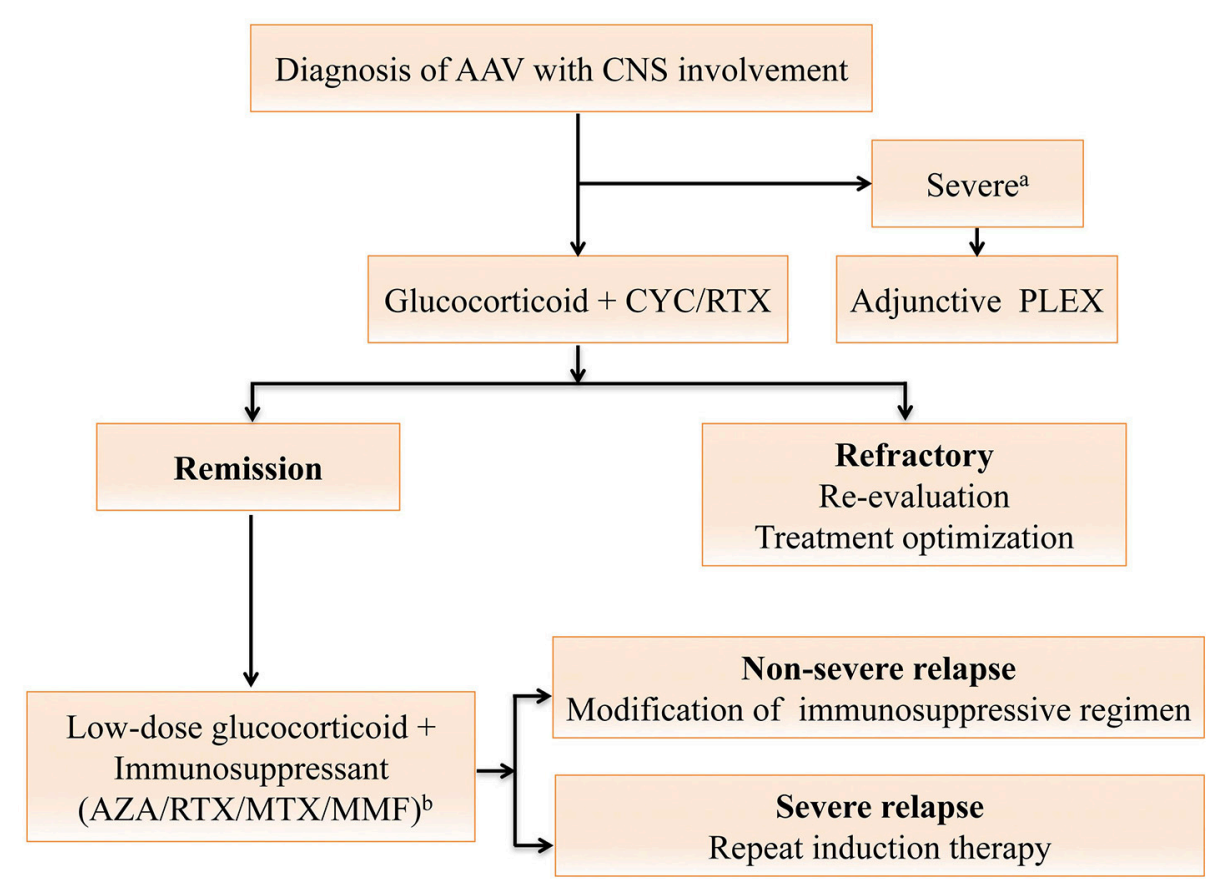

FIGURE 2 | Treatment algorithm for anti-neutrophil cytoplasmic antibody (ANCA)-associated vasculitides (AAV) with central nervous system (CNS) involvement. The algorithm is formulated and terms are defined according to the 2016 European league Against Rheumatism (EULAR)/European Renal Association-European Dialysis and Transplant Association (ERA-EDTA) recommendations (61). For AAV with CNS involvement, the remission-induction therapy mainly consists of high-dose steroids and cyclophosphamide (CYC), or rituximab (RTX) for CYC-intolerant patients. Plasma exchange should be considered for those with a serum creatine level of $\geq 500$ $\mu \mathrm{mol} / \mathrm{L}$ or diffuse alveolar hemorrhage. Once complete remission is achieved, patients should be switched to the maintenance regimen. A combination of low-dose steroid and an oral immunosuppressive agent including azathioprine (AZA), methotrexate (MTX), mycophenolate mofetil (MMF) or RTX is used for at least 24 months (61). For patients refractory to the remission-induction therapy, referral to experts for reevaluation and treatment optimization is warranted (61). Severe relapses with organ- or life-threatening conditions are treated as per new disease, while non-severe relapses are managed with modification of the previous immunosuppressive regimen (61). ${ }^{\mathrm{a}} \mathrm{AAV}$ with severe renal impairment or diffuse alveolar hemorrhage; ${ }^{\mathrm{b}}$ Drugs are listed in order of the strength of vote. AAV, anti-neutrophil cytoplasmic antibody-associated vasculitides; ANCA, anti-neutrophil cytoplasmic antibodies; AZA, azathioprine; CNS, central nervous system; CYC, cyclophosphamide; MTX, methotrexate; MMF, mycophenolate mofetil; PLEX, plasma exchange; RTX, rituximab.

patient, as per the European Renal Association-European Dialysis and Transplant Association (ERA-EDTA) management recommendations for AAV (61). Generally, CNS involvement is regarded as an organ-threatening manifestation in $\mathrm{AAV}$, especially for those with meningeal inflammation or retro-orbital disease. Remission-induction treatment for organ-threatening AAV typically consists of high-dose glucocorticoids and oral or intravenous (IV) cyclophosphamide (CYC) (1), termed CYCbased therapy. Glucocorticoids usually start with the dose of $1 \mathrm{~g}$ intravenous methylprednisolone on 3 consecutive days, followed with $1 \mathrm{mg} / \mathrm{kg}$ daily oral corticosteroids (up to $80 \mathrm{mg}$ per day), and a gradual reduction to daily dose of 7.5 to $10 \mathrm{mg}$ within 35 months $(7,10)$. CYC can be given either intravenously in pulses or orally. The intravenous CYC, at a dose of $15 \mathrm{mg} / \mathrm{kg}$ (up to $1200 \mathrm{mg}$ ), are prescribed every 2 weeks initially and every 3 weeks from the 4 th pulse. Daily oral CYC is given at a dose of $2 \mathrm{mg} / \mathrm{kg} / \mathrm{d}$. The CYC-based therapy is effective in $70-90 \%$ of patients (70), and the oral regimen can better prevent relapses (71). However, the daily oral regimen, compared with intravenous CYC, also poses more safety issues due to the cumulative toxicity (71, 72) including infertility, bladder hemorrhage, severe cytopenias, serious infection, and an increased risk of malignancy. Apart from the most recognized CYC-based therapy, the identification of the important role of $\mathrm{B}$ cells in the pathogenesis of AAV facilitated the use of rituximab (RTX) as an alternative to CYC (73). Two large randomized trials revealed RTX $\left(375 \mathrm{mg} / \mathrm{m}^{2}\right.$, once a week for four infusions) to be equivalent to CYC in terms of efficacy and safety in remission induction for AAV among treatment-naïve patients, and likely superior for relapsing disease (74).

Once complete remission (absence of any disease activity, usually attained after 8-12 weeks of treatment) is achieved, patients should be started with maintenance therapy to prevent further relapses, which should be continued for at least 24 months (61). In the remission maintenance phase, the induction regimen is switched to a combination of low-dose glucocorticoids and an oral immunosuppressive agent such as azathioprine (AZA), methotrexate (MTX), mycophenolate mofetil (MMF), or RTX (61). AZA (2 mg/kg/day) is the most commonly used immunosuppressive agent for remission maintenance, with MTX (20-25 mg/kg/week) as a similar alternative (72). AZA, with a lower relapse rate, is generally preferred over MMF. However, the relapse rate remains as high as $40 \%$ by 2 years despite the maintenance therapy with AZA or MTX (75). RTX is a 
potentially safer maintenance drug with a higher efficacy (76). However, the utility and toxicity profile of RTX require further confirmation (76-80). In addition, the adjunctive use of plasma exchange (PLEX) (7 sessions over 2 weeks) in AAV patients with severe renal dysfunction and/or alveolar hemorrhage seems reasonable in the short-term, but remains elusive in the long run (81).

Another issue to tackle in AAV is the treatment response. Refractory to remission-induction treatment is defined as follows: (1) unchanged or increased disease activity after 4 weeks of treatment (2) $<50 \%$ reduction in the disease score [e.g., Birmingham Vasculitis Activity Score (BVAS)] after 6 weeks of treatment (3) Presence of at least one major or three minor items on the disease activity score after over 12 weeks of treatment $(61,82)$. For AAV patients refractory to therapy, reevaluation and optimization of treatment, in close collaboration with experts, are recommended (61). Adjunctive intravenous immunoglobulin may help for those with persistent low disease activity $(61,83)$. Furthermore, relapses are not uncommon in AAV. Major relapses with organ- or life-threatening conditions are treated as per new disease, while non-severe relapses should be managed with an escalation or modification of the previous immunosuppressive regimen (61).

\section{CONCLUSION AND FUTURE PERSPECTIVES}

For neurologists, prompt diagnosis of AAV in patients with CNS presentations allows timely treatment and thus a dramatic improvement in prognosis. A myriad of CNS presentations including hypertrophic pachymeningitis, ischemic stroke, intracranial hemorrhage and pituitary dysfunction, combined

\section{REFERENCES}

1. Jennette JC, Falk RJ, Bacon PA, Basu N, Cid MC, Ferrario F, et al. 2012 revised International Chapel Hill consensus conference nomenclature of vasculitides. Arthritis Rheum. (2013) 65:1-11. doi: 10.1002/art.37715

2. Sada K, Yamamura M, Harigai M, Fujii T, Dobashi H, Takasaki $\mathrm{Y}$, et al. Classification and characteristics of Japanese patients with antineutrophil cytoplasmic antibody-associated vasculitis in a nationwide prospective, inception cohort study. Arthritis Res Ther. (2014) 16: R101. doi: 10.1186/ar4550

3. Zhang W, Zhou G, Shi Q, Zhang X, Zeng XF, Zhang FC. Clinical analysis of nervous system involvement in ANCA-associated systemic vasculitides. Clin Exp Rheumatol. (2009) 27(1 Suppl 52):S65-9.

4. Agard C, Mouthon L, Mahr A, Guillevin Lc. Microscopic polyangiitis and polyarteritis nodosa: how and when do they start? Arthrit Rheum. (2003) 49:709-15. doi: 10.1002/art.11387

5. Graf J. Central nervous system disease in antineutrophil cytoplasmic antibodies-associated vasculitis. Rheum Dis Clin North Am. (2017) 43:573-8. doi: 10.1016/j.rdc.2017.06.006

6. Bossuyt X, Cohen Tervaert JW, Arimura Y, Blockmans D, FloresSuarez LF, Guillevin L, et al. Position paper: Revised 2017 international consensus on testing of ANCAs in granulomatosis with polyangiitis and microscopic polyangiitis. Nat Rev Rheumatol. (2017) 13:683-92. doi: 10.1038/nrrheum.2017.140

7. Kallenberg CG. Key advances in the clinical approach to ANCAassociated vasculitis. Nat Rev Rheumatol. (2014) 10:484-93. doi: 10.1038/nrrheum.2014.104 with certain systemic symptoms, raise the suspicion of AAV. Further ancillary tests are required, among which ANCA testing yields a great diagnostic value. ANCA positivity strongly suggests the diagnosis of AAV but ANCA negativity does not rule out the diagnosis. Once diagnosed, early treatment with steroid and immunosuppressant is essential to prevent neurological relapses and sequelae.

Recent evidence suggests the presence of "CNS-limited AAV" as a distinct subset in AAV. Efforts to better elucidate its phenotypic features, optimal treatment and long-term outcome are an important focus of future research. Furthermore, accumulating studies suggest that PR3-ANCAs and MPOANCAs define distinctive conditions among patients with AAV. Whether the same rule applies to the neurological conditions in CNS-involved AAV, however, remains unknown. Continued attempts are needed to validate the utility of ANCA specificity in classifying CNS manifestations, guiding treatment decisions, and predicting prognosis.

\section{AUTHOR CONTRIBUTIONS}

YZ: contributed to data collection, conception of the work and drafting the manuscript. YxZ: conception of the work and revising the manuscript. MC: revising the manuscript and figures. NL: revising the manuscript and tables. $\mathrm{ZC}$ and $\mathrm{MD}$ : revising the manuscript and final approval of the version to be published.

\section{FUNDING}

This work was supported by the National Natural Science Foundation of China (grant numbers $81671283 ; 81872844$ ).

8. Lyons PA, Rayner TF, Trivedi S, Holle JU, Watts RA, Jayne DR, et al. Genetically distinct subsets within ANCA-associated vasculitis. $N$ Engl J Med. (2012) 367:214-23. doi: 10.1056/NEJMoa1108735

9. van der Geest KSM, Brouwer E, Sanders JS, Sandovici M, Bos NA, Boots AMH, et al. Towards precision medicine in ANCA-associated vasculitis. Rheumatology (2018) 57:1332-9. doi: 10.1093/rheumatology/ $\operatorname{kex} 367$

10. Sznajd J, Mukhtyar C. How to treat ANCAassociated vasculitis: practical messages from 2016 EULAR/ERAEDTA recommendations. Pol Arch Med Wewn (2016) 126:781-8. doi: 10.20452/pamw.3598

11. Jennette JC, Falk RJ. Pathogenesis of antineutrophil cytoplasmic autoantibody-mediated disease. Nat Rev Rheumatol. (2014) 10:463-73. doi: 10.1038/nrrheum.2014.103

12. Cornec D, Gall EC-L, Fervenza FC, Specks U. ANCA-associated vasculitis - clinical utility of using ANCA specificity to classify patients. Nat Rev Rheumatol. (2016) 12:570-9. doi: 10.1038/nrrheum.2016.123

13. Chen M, Jayne DRW, Zhao M-H. Complement in ANCA-associated vasculitis: mechanisms and implications for management. Nat Rev Nephrol. (2017) 13:359-67. doi: 10.1038/nrneph.2017.37

14. Soderberg D, Segelmark M. Neutrophil extracellular traps in ANCA-Associated Vasculitis. Front Immunol. (2016) 7:256. doi: 10.3389 /fimmu. 2016.00256

15. Dumoitier N, Terrier B, London J, Lofek S, Mouthon L. Implication of B lymphocytes in the pathogenesis of ANCA-associated vasculitides. Autoimmun Rev. (2015) 14:996-1004. doi: 10.1016/j.autrev.2015.06.008

16. Feng B, Tang Y, Chen B, Xu C, Wang Y, Dai Y, et al. Transient increase of interleukin-1beta after prolonged febrile seizures promotes 
adult epileptogenesis through long-lasting upregulating endocannabinoid signaling. Sci Rep. (2016) 6:21931. doi: 10.1038/srep21931

17. Zhao J, Wang Y, Xu C, Liu K, Wang Y, Chen L, et al. Therapeutic potential of an anti-high mobility group box-1 monoclonal antibody in epilepsy. Brain Behav Immun. (2017) 64:308-19. doi: 10.1016/j.bbi.2017.02.002

18. Crisp SJ, Kullmann DM, Vincent A. Autoimmune synaptopathies. Nat Rev Neurosci. (2016) 17:103-17. doi: 10.1038/nrn.2015.27

19. Berlit P. Diagnosis and treatment of cerebral vasculitis. Ther Adv Neurol Disord. (2010) 3:29-42. doi: 10.1177/1756285609347123

20. Morgan MD, Day CJ, Piper KP, Khan N, Harper L, Moss PA, et al. Patients with Wegener's granulomatosis demonstrate a relative deficiency and functional impairment of T-regulatory cells. Immunology (2010) 130:64-73. doi: 10.1111/j.1365-2567.2009.03213.x

21. Petersen HJ, Smith AM. The role of the innate immune system in granulomatous disorders. Front Immunol. (2013) 4:120. doi: 10.3389 /fimmu. 2013.00120

22. Holle JU, Gross WL. Neurological involvement in Wegener's granulomatosis. Curr Opin Rheumatol. (2011) 23:7-11. doi: 10.1097/BOR.0b013e3283 $4115 \mathrm{f} 9$

23. Drachman DA. Neurological Complications of Wegener's Granulomatosis. Arch Neurol. (1963) 8:145-55. doi: 10.1001/archneur.1963.00460020045003

24. Yokoseki A, Saji E, Arakawa M, Kosaka T, Hokari M, Toyoshima $\mathrm{Y}$, et al. Hypertrophic pachymeningitis: significance of myeloperoxidase anti-neutrophil cytoplasmic antibody. Brain (2014) 137(Pt 2):520-36. doi: 10.1093/brain/awt314

25. Fragoulis GE, Lionaki S, Venetsanopoulou A, Vlachoyiannopoulos PG, Moutsopoulos HM, Tzioufas AG. Central nervous system involvement in patients with granulomatosis with polyangiitis: a single-center retrospective study. Clin Rheumatol. (2018) 37:737-47. doi: 10.1007/s10067-017-3835-y

26. Seror R, Mahr A, Ramanoelina J, Pagnoux C, Cohen P, Guillevin L. Central nervous system involvement in Wegener granulomatosis. Medicine (2006) 85:54-65. doi: 10.1097/01.md.0000200166.90373.41

27. Weiner M, Segelmark M. The clinical presentation and therapy of diseases related to anti-neutrophil cytoplasmic antibodies (ANCA). Autoimmun Rev. (2016) 15:978-82. doi: 10.1016/j.autrev.2016.07.016

28. Watts R, Lane S, Hanslik T, Hauser T, Hellmich B, Koldingsnes W, et al. Development and validation of a consensus methodology for the classification of the ANCA-associated vasculitides and polyarteritis nodosa for epidemiological studies. Ann Dis. (2006) 66:222-7. doi: 10.1136/ard.2006.054593

29. Yanagiha K, Ishii K, Ueno T, Marushima A, Tamaoka A. Medial medullary infarction caused by antineutrophil cytoplasmic antibody-related vasculitis: Case report and review of the literature. Medicine (2017) 96:e7722. doi: 10.1097/MD.0000000000007722

30. Chen NC, Lai PH, Fang HC, Chou KJ, Chen CL. Microscopic polyangiitis with an initial presentation of pontine infarction. Am J Med Sci. (2012) 344:163-5. doi: 10.1097/MAJ.0b013e3182536789

31. Iglesias E, Eleftheriou D, Mankad K, Prabhakar P, Brogan PA. Microscopic polyangitis presenting with hemorrhagic stroke. J Child Neurol. (2014) 29:NP1-4. doi: 10.1177/0883073813488661

32. Ito $\mathrm{Y}$, Suzuki $\mathrm{K}$, Yamazaki $\mathrm{T}$, Yoshizawa $\mathrm{T}$, Ohkoshi $\mathrm{N}$, Matsumura A. ANCA-associated vasculitis (AAV) causing bilateral cerebral infarction and subsequent intracerebral hemorrhage without renal and respiratory dysfunction. J Neurol Sci. (2006) 240(1-2):99-101. doi: $10.1016 /$ j.jns.2005.07.003

33. Aratani S, Sakai Y, Tsuruoka S. A case of microscopic polyangiitis with subarachnoid hemorrhage and cardiovascular complications. J Nippon Med Sch. (2017) 84:251-5. doi: 10.1272/jnms.84.251

34. Abdel Razek AA, Alvarez H, Bagg S, Refaat S, Castillo M. Imaging spectrum of CNS vasculitis. Radiographics (2014) 34:873-94. doi: 10.1148/rg.344135028

35. Fuentes AG, Komarla A, Gomez JI. Posterior reversible encephalopathy syndrome in a patient with ANCA-associated vasculitis. Rheumatol Int. (2012) 32:2529-30. doi: 10.1007/s00296-010-1367-8

36. Patel UV, Patel NJ. Posterior reversible leukoencephalopathy syndrome as a presenting manifestation of p-ANCA-associated vasculitis. BMJ Case Rep. (2014) 2014:bcr2013202022. doi: 10.1136/bcr-2013-202022

37. De Luna G, Terrier B, Kaminsky P, Le Quellec A, Maurier F, Solans R, et al. Central nervous system involvement of granulomatosis with polyangiitis: clinical-radiological presentation distinguishes different outcomes. Rheumatology (2015) 54:424-32. doi: 10.1093/rheumatology/keu336

38. Spisek R, Kolouchova E, Jensovsky J, Rusina R, Fendrych P, Plas $\mathrm{J}$, et al. Combined CNS and pituitary involvement as a primary manifestation of Wegener granulomatosis. Clin Rheumatol. (2006) 25:739-42. doi: 10.1007/s10067-005-0065-5

39. Berlis A, Petschner F, Botefur IC, Spreer J. Wegener granuloma in the fourth ventricle. AJNR Am J Neuroradiol. (2003) 24:523-5.

40. Mattioli F, Capra R, Rovaris M, Chiari S, Codella M, Miozzo A, et al. Frequency and patterns of subclinical cognitive impairment in patients with ANCA-associated small vessel vasculitides. J Neurol Sci. (2002) 195:161-6. doi: 10.1016/S0022-510X(02)00015-1

41. Yonekawa $\mathrm{T}$, Murai $\mathrm{H}$, Utsuki S, Matsushita T, Masaki K, Isobe N, et al. A nationwide survey of hypertrophic pachymeningitis in Japan. J Neurol Neurosurg Psychiatry (2013) 85:732-9. doi: 10.1136/jnnp-2013306410

42. Choi HA, Lee MJ, Chung CS. Characteristics of hypertrophic pachymeningitis in patients with granulomatosis with polyangiitis. J Neurol. (2017) 264:724-32. doi: 10.1007/s00415-017-8416-0

43. Pakalniskis MG, Berg AD, Policeni BA, Gentry LR, Sato Y, Moritani T, et al. The many faces of granulomatosis with polyangiitis: a review of the head and neck imaging manifestations. Am J Roentgenol. (2015) 205:W619-29. doi: 10.2214/AJR.14.13864

44. Kapoor E, Cartin-Ceba R, Specks U, Leavitt J, Erickson B, Erickson D. Pituitary dysfunction in granulomatosis with polyangiitis: the mayo clinic experience. $J$ Clin Endocrinol Metab. (2014) 99:3988-94. doi: 10.1210/jc.2014-1962

45. Esposito D, Trimpou P, Giugliano D, Dehlin M, Ragnarsson O. Pituitary dysfunction in granulomatosis with polyangiitis. Pituitary (2017) 20:594-601. doi: 10.1007/s11102-017-0811-0

46. Yong TY, Li JY. Pituitary involvement in granulomatosis with polyangiitis. $J$ Clin Rheumatol. (2014) 20:123-4. doi: 10.1097/RHU.0000000000000092

47. De Parisot A, Puechal X, Langrand C, Raverot G, Gil H, Perard L, et al. Pituitary involvement in granulomatosis with polyangiitis: report of 9 patients and review of the literature. Medicine (2015) 94:e748. doi: 10.1097/MD.0000000000000748

48. Decker ML, Emery DJ, Smyth PS, Lu J-Q, Lacson A, Yacyshyn E. Microscopic polyangiitis with spinal cord involvement: a case report and review of the literature. J Stroke Cereb Dis. (2016) 25:1696-704. doi: 10.1016/j.jstrokecerebrovasdis.2016.01.034

49. Nair RP, Kongwad LI. Nontuberculous antineutrophil cytoplasmic antibodypositive pachymeningitis presenting as high cervical myelopathy. World Neurosurg. (2017) 107:1042 e1- 1042.e5. doi: 10.1016/j.wneu.2017.08.035

50. Soderstrom A, Revaz S, Dudler J. Cranial neuropathies in granulomatosis with polyangiitis (Wegener's): a case-based review. Clin Rheumatol. (2015) 34:591-6. doi: 10.1007/s10067-013-2420-2

51. Gwathmey KG, Burns TM, Collins MP, Dyck PJ. Vasculitic neuropathies. Lancet Neurol. (2014) 13:67-82. doi: 10.1016/S1474-4422(13)70236-9

52. Guillevin L, Durand-Gasselin B, Cevallos R, Gayraud M, Lhote F, Callard P, et al. Microscopic polyangiitis: clinical and laboratory findings in eighty-five patients. Arthritis Rheum. (1999) 42:421-30.

53. Nowack R, Wachtler P, Kunz J, Rasmussen N. Cranial nerve palsy in Wegener's granulomatosis-lessons from clinical cases. J Neurol. (2009) 256:299-304. doi: 10.1007/s00415-009-0121-1

54. Kubaisi B, Abu Samra K, Foster CS. Granulomatosis with polyangiitis (Wegener's disease): An updated review of ocular disease manifestations. Intractable Rare Dis Res. (2016) 5:61-9. doi: 10.5582/irdr.2016.01014

55. Papaliodis GN. Ophthalmologic manifestations of systemic vasculitis. Curr Opin Ophthalmol. (2017) 28:613-6. doi: 10.1097/ICU.0000000000000422

56. Vodopivec I, Lobo AM, Prasad S. Ocular inflammation in neurorheumatic disease. Semin Neurol. (2014) 34:444-57. doi: 10.1055/s-0034-1390393

57. Hilhorst M, van Paassen P, Tervaert JW, Limburg Renal R. Proteinase 3-ANCA Vasculitis versus Myeloperoxidase-ANCA Vasculitis. J Am Soc Nephrol. (2015) 26:2314-27. doi: 10.1681/ASN.2014090903

58. Lionaki S, Blyth ER, Hogan SL, Hu Y, Senior BA, Jennette CE, et al. Classification of antineutrophil cytoplasmic autoantibody vasculitides: the role of antineutrophil cytoplasmic autoantibody specificity for myeloperoxidase or proteinase 3 in disease recognition and prognosis. Arthritis Rheum. (2012) 64:3452-62. doi: 10.1002/art.34562 
59. Mahr A, Katsahian S, Varet H, Guillevin L, Hagen EC, Hoglund $\mathrm{P}$, et al. Revisiting the classification of clinical phenotypes of antineutrophil cytoplasmic antibody-associated vasculitis: a cluster analysis. Ann Rheum Dis. (2013) 72:1003-10. doi: 10.1136/annrheumdis-2012201750

60. Unizony S, Villarreal M, Miloslavsky EM, Lu N, Merkel PA, Spiera R, et al. Clinical outcomes of treatment of anti-neutrophil cytoplasmic antibody (ANCA)-associated vasculitis based on ANCA type. Ann Rheum Dis. (2016) 75:1166-9. doi: 10.1136/annrheumdis-2015208073

61. Yates M, Watts RA, Bajema IM, Cid MC, Crestani B, Hauser T, et al. EULAR/ERA-EDTA recommendations for the management of ANCA-associated vasculitis. Ann Rheum Dis. (2016) 75:1583-94. doi: 10.1136/annrheumdis-2016-209133

62. Basu N, Watts R, Bajema I, Baslund B, Bley T, Boers M, et al. EULAR points to consider in the development of classification and diagnostic criteria in systemic vasculitis. Ann Rheum Dis. (2010) 69:1744-50. doi: 10.1136/ard.2009.119032

63. Wiik AS. Autoantibodies in ANCA-associated vasculitis. Rheum Dis Clin North Am. (2010) 36:479-89. doi: 10.1016/j.rdc.2010.05.001

64. Csernok E, Moosig F. Current and emerging techniques for ANCA detection in vasculitis. Nat Rev Rheumatol. (2014) 10:494-501. doi: 10.1038/nrrheum.2014.78

65. Segelmark M, Phillips BD, Hogan SL, Falk RJ, Jennette JC. Monitoring proteinase 3 antineutrophil cytoplasmic antibodies for detection of relapses in small vessel vasculitis. Clin Diagn Lab Immunol. (2003) 10:769-74. doi: 10.1128/CDLI.10.5.769-774.2003

66. Tomasson G, Grayson PC, Mahr AD, Lavalley M, Merkel PA. Value of ANCA measurements during remission to predict a relapse of ANCA-associated vasculitis-a meta-analysis. Rheumatology (2012) 51:100-9. doi: 10.1093/rheumatology/ker280

67. Morgan MD, Szeto M, Walsh M, Jayne D, Westman K, Rasmussen N, et al. Negative anti-neutrophil cytoplasm antibody at switch to maintenance therapy is associated with a reduced risk of relapse. Arthritis Res Ther. (2017) 19:129. doi: 10.1186/s13075-017-1321-1

68. Kemna MJ, Damoiseaux J, Austen J, Winkens B, Peters J, van Paassen P, et al. ANCA as a predictor of relapse: useful in patients with renal involvement but not in patients with nonrenal disease. J Am Soc Nephrol. (2015) 26:537-42. doi: 10.1681/ASN.2013111233

69. Specks U. Accurate relapse prediction in ANCA-associated vasculitisthe search for the Holy Grail. J Am Soc Nephrol. (2015) 26:505-7. doi: 10.1681/ASN.2014080817

70. Jones RB, Tervaert JW, Hauser T, Luqmani R, Morgan MD, Peh CA, et al. Rituximab versus cyclophosphamide in ANCA-associated renal vasculitis. $N$ Engl J Med. (2010) 363:211-20. doi: 10.1056/NEJMoa0909169

71. Harper L, Morgan MD, Walsh M, Hoglund P, Westman K, Flossmann O, et al. Pulse versus daily oral cyclophosphamide for induction of remission in ANCA-associated vasculitis: long-term follow-up. Ann Rheum Dis. (2012) 71:955-60. doi: 10.1136/annrheumdis-2011-200477

72. Pagnoux C, Mahr A, Hamidou MA, Boffa JJ, Ruivard M, Ducroix JP, et al. Azathioprine or Methotrexate Maintenance for ANCA-Associated
Vasculitis. N Engl J Med. (2008) 359:2790-803. doi: 10.1056/NEJMoa08 02311

73. Jennette JC, Falk RJ. B cell-mediated pathogenesis of ANCAmediated vasculitis. Semin Immunopathol. (2014) 36:327-38. doi: 10.1007/s00281-014-0431-y

74. Stone JH, Merkel PA, Spiera R, Seo P, Langford CA, Hoffman GS, et al. Rituximab versus cyclophosphamide for ANCA-associated vasculitis. N Engl J Med. (2010) 363:221-32. doi: 10.1056/NEJMoa0909905

75. Tanna A, Pusey C. Clinical trials. Rituximab for maintenance of remission in AAV. Nat Rev Nephrol. (2015) 11:131-2. doi: 10.1038/nrneph.2014.254

76. Pendergraft WF, Cortazar FB, Wenger J, Murphy AP, Rhee EP, Laliberte KA, et al. Long-term maintenance therapy using rituximab-induced continuous Bcell depletion in patients with ANCA vasculitis. Clin J Am Soc Nephrol. (2014) 9:736-44. doi: 10.2215/CJN.07340713

77. Cartin-Ceba R, Golbin JM, Keogh KA, Peikert T, Sanchez-Menendez M, Ytterberg SR, et al. Rituximab for remission induction and maintenance in refractory granulomatosis with polyangiitis (Wegener's): ten-year experience at a single center. Arthritis Rheum. (2012) 64:3770-8. doi: 10.1002/art.34584

78. Rhee EP, Laliberte KA, Niles JL. Rituximab as maintenance therapy for antineutrophil cytoplasmic antibody-associated vasculitis. Clin J Am Soc Nephrol. (2010) 5:1394-400. doi: 10.2215/CJN.08821209

79. Besada E, Koldingsnes W, Nossent JC. Long-term efficacy and safety of pre-emptive maintenance therapy with rituximab in granulomatosis with polyangiitis: results from a single centre. Rheumatology (2013) 52:2041-7. doi: 10.1093/rheumatology/ket257

80. Guillevin L, Pagnoux C, Karras A, Khouatra C, Aumaitre O, Cohen P, et al. Rituximab versus azathioprine for maintenance in ANCA-associated vasculitis. N Engl J Med. (2014) 371:1771-80. doi: 10.1056/NEJMoa1404231

81. Matthew J. Koster KJW. Recent advances in understanding and treating vasculitis. F1000Research (2016) 5:1436. doi: 10.12688/f1000research. 8403.1

82. Hellmich B, Flossmann O, Gross WL, Bacon P, Cohen-Tervaert JW, Guillevin L, et al. EULAR recommendations for conducting clinical studies and/or clinical trials in systemic vasculitis: focus on anti-neutrophil cytoplasm antibody-associated vasculitis. Ann Rheum Dis. (2007) 66:605-17. doi: 10.1136/ard.2006.062711

83. Fortin PM, Tejani AM, Bassett K, Musini VM. Intravenous immunoglobulin as adjuvant therapy for Wegener's granulomatosis. Cochrane Database Syst Rev. (2013) CD007057. doi: 10.1002/14651858.CD007057

Conflict of Interest Statement: The authors declare that the research was conducted in the absence of any commercial or financial relationships that could be construed as a potential conflict of interest.

Copyright (C) 2019 Zheng, Zhang, Cai, Lai, Chen and Ding. This is an open-access article distributed under the terms of the Creative Commons Attribution License (CC $B Y)$. The use, distribution or reproduction in other forums is permitted, provided the original author(s) and the copyright owner(s) are credited and that the original publication in this journal is cited, in accordance with accepted academic practice. No use, distribution or reproduction is permitted which does not comply with these terms. 